\title{
Servicio/trabajo social con niños y niñas con necesidades educativas especiales en España
}

\author{
Service/social work with children and girls with special educational \\ needs in Spain
}

\section{Serviço/trabalho social com meninos e meninas com necessidades educativas especiais na Espanha}

\author{
Enrique Pastor Seller \\ Universidad de Murcia, Trabajo Social y Servicios Sociales, Profesor e Investigador \\ Titular
}

https://orcid.org/0000-0001-8693-5138

\section{Carla Grau Bastida}

Universidad de Murcia, Departamento Trabajo Social y Servicios Sociale, Colaboradora https://orcid.org/0000-0001-6390-1453

Resumen: El articulo presente la realidad del Servicio /Trabajo Social en el ámbito educativo en España respecto de los centros/servicios en los que se integra, necesidades que atiende, funciones desempeñadas y expectativas de futuro. Para la consecución de los objetivos previstos se ha realizado una investigación empírica con una metodología mixta (cuantitativa y cualitativa), mediante el análisis documental, la administración de cuestionarios a profesionales, la realización de entrevistas individuales y grupales a expertos. En la investigación han participado un total de 30 profesionales del Trabajo/ Servicio Social que desempeñan su labor en el ámbito educativo en el sureste de España (provincias de Almería, Alicante y Murcia). Los resultados alcanzados permiten aproximarnos a la realidad del Trabajo/ Servicio Social en el ámbito educativo en el Sureste de España. De la investigación se constata que el perfil profesional se caracteriza por una feminización de la población con un edad promedio de entre 31 y 51 años; los centros y servicios en los que se integran son en su mayoría de dependencia pública, principalmente en Equipos de Orientación Educativa; atienden necesidades como el absentismo escolar, orientación familiar, atención a la diversidad y necesidades educativas especiales; sus funciones van destinadas a prevenir y atender problemáticas individuales de los/as alumnos/as, en relación a los contexto familiar y escolar; existe una escasa presencia de trabajadores/as sociales en el sistema educativo, asi como una falta de reconocimiento de esta figura profesional en educación.

Palabras clave: Servicio/Trabajo social educativo. Funciones. Necesidades Atendidas. Expectativas profesionales.

Doctor en Sociologia por la Universidad de Murcia (España).

Graduada en Trabajo Social por la Universidad de Murcia (España). 
Abstract: The article presents the reality of the Service/Social Work in the educational field in Spain with respect to the centers/services in which it is integrated, needs it serves, functions performed and future expectations. In order to achieve the planned objectives, an empirical research was carried out with a mixed methodology (quantitative and qualitative), through document analysis, the administration of questionnaires to professionals, the conduct of individual and group interviews with experts. A total of 30 Labor/Social Service professionals participated in the research and work in the educational field in the southeast of Spain (provinces of Almeria, Alicante and Murcia). The results achieved allow us to approach the reality of the Work I Social Service in the educational field in the Southeast of Spain. The research shows that the professional profile is characterized by a feminization of the population with an average age of between 31 and 51 years; the centers and services in which they are integrated are in their majority of public dependence, mainly in Educational Guidance Teams; they attend needs such as school absenteeism, family orientation, attention to diversity and special educational needs; its functions are intended to prevent and address individual problems of the students, in relation to the family and school context; There is a scarce presence of social workers in the education system, as well as a lack of recognition of this professional figure in education.

Keywords: Educational social work/service. Functions. Needs Addressed. Professional expectations.

Resumo: 0 artigo apresenta a realidade do Serviço/Trabalho Social na área educacional da Espanha no que diz respeito aos centros/serviços que os integram, as necessidades que atendem, as funções desempenhadas e as expectativas de futuro. Para atingir os objetivos planejados, foi realizada uma pesquisa empírica com metodologia mista (quantitativa e qualitativa), por meio de análise documental, aplicação de questionários e a realização de entrevistas individuais e coletivas com especialistas. Participaram da pesquisa um total de 30 profissionais do Serviço/Trabalho Social que desempenham suas atividades no campo educacional no sudeste da Espanha Imunicípios de Almería, Alicante e Múrcia). Os resultados alcançados nos permitem aproximar da realidade do Serviço/Trabalho Social, no campo educacional revelando um perfil profissional caracterizado pela feminização, com idade média entre 31 e 51 anos; os centros e serviços nos quais estes profissionais estão integrados são, em sua maioria, de dependência pública, principalmente as equipes de orientação educacional; atendem necessidades como evasão escolar, orientação familiar, atenção à diversidade e necessidades educacionais especiais; suas funções visam prevenir e abordar problemas individuais dos alunos, em relação ao contexto familiar e escolar; há uma escassa presença de assistentes sociais no sistema educacional, bem como a falta de reconhecimento dessa figura profissional na educação.

Palavras-chave: Serviço/Trabalho social educativo. Função. Necessidades atendidas. Expectativas profissionais.

Recibido en 24 de junio de 2018 Acepto en 6 de febrero de 2019 Publicado el 14 de mayo de 2019 


\section{INTRODUCCIÓN}

La investigación que orienta el presente artículo surge de los interrogantes planteados en lo relativo a la realidad del Servicio Social en el ámbito educativo: perfil profesional del trabajador/a social, centros y servicios en los que se integran, funciones que desempeñan, así como las oportunidades de empleabilidad en el Sistema Educativo. El artículo presenta los resultados derivados de una investigación empírica realizada orientada a conocer $y$ analizar las principales funciones que estos actores sociales desempeñan en el ámbito educativo y las necesidades educativas que presentan los/as niños $/ a^{3}{ }^{3}$ que son atendidos en dicho servicio.

El sistema educativo español ha sufrido profundas y continuas modificaciones normativas, competenciales y organizacionales, en razón de las realidades de nuestro país como Estado democrático y de derecho en el marco de la integración europea y la globalización. De esta forma, los diferentes cambios politicos, económicos y sociales que afectan al sistema educativo en España, provocan que las diferentes normativas estatales y autonómicas de educación hayan ido modificándose. Así, la actual Ley para la Mejora de la Calidad Educativa (ESPAÑA, 2013), hace especial referencia a la necesidad de adaptar la educación a las necesidades de la sociedad en general y a la diversidad del alumnado en particular, con el objetivo de conseguir la inclusión social de los/as niños/as.

Partiendo de la base de la igualdad de oportunidades y de la universalidad de la que está dotada la educación, se ha de tomar como núcleo, origen y fin de este ámbito a las personas destinatarias: los/as alumnos/as. Por consiguiente, se ha de tener presente en todo momento la consideración inexorable de la diversidad del alumnado, de las diferentes necesidades y problemáticas que presentan y, con ello, la preocupación imperante por el conjunto de actores sociales que se integran en los ámbitos socioeducativos en procurar una educación de calidad y que mediante ésta se logre el máximo desarrollo de las capacidades del alumnado y su inclusión social (PICORNELL; PASTOR, 2016). De ahi la necesidad de conocer y analizar el papel que para dicho fin desempeña el profesional de Trabajo Social en el ámbito educativo.

La figura profesional del Trabajo Social se incorporó al sistema educativo en los años 70, junto a otros perfiles profesionales no docentes, con el fin de atender las nuevas necesidades del alumnado, pretendiendo la protección a los/as niños/as, en especial a aquellos con necesidades educativas especiales. La Ley General de Educación y Financiamiento de la Reforma Educativa, promulgada el 4 de agosto de 1970, incluyó la Educación Especial como modalidad del Sistema Educativo en los centros escolares ordinarios,

\footnotetext{
Se utiliza el término 'niño', en referencia a la Convención de Derechos del Niño (1989), para designar a todo ser humano menor de 18 años; entendiendo niño, niña y adolescente.
} 
ya que esta normativa registra el concepto de integración escolar mediante Aulas de Educación Especial. La Constitución Española (ESPAÑA, 1978), en su artículo 27, reconoce el derecho a la educación, la obligatoriedad y gratuidad de la enseñanza básica, así como la libertad de enseñanza y la autonomía universitaria, entre otros, derechos. Asimismo, establece las bases de la descentralización de las competencias en materia de educación entre Administración central y las Comunidades Autónomas.

La Ley Orgánica 8/1985, de 3 de julio, reguladora del Derecho a la Educación (ESPAÑA, 1985, p. 21016), se encargó de establecer que "todos los españoles tienen derecho a una educación básica que les permita el desarrollo de su propia personalidad y la realización de una actividad útil a la sociedad." De esta forma se racionaliza y planifica la enseñanza considerando los principios de participación, igualdad y pluralismo ya recogidos en la Constitución Española, para el ámbito educativo. En esta misma línea cabe mencionar la Ley 12/1987, de 2 de julio (ESPAÑA, 1987), sobre establecimiento de gratuidad de los estudios de Bachillerato, Formación Profesional y Artes Aplicadas y Oficios Artísticos en los Centros públicos y la autonomía de gestión económica de los Centros docentes públicos no universitarios.

El lnstituto Nacional de Educación Especial, consolidó el Plan Nacional de Educación Especial, por el que se establecieron Equipos Multiprofesionales que tenían como finalidad la evaluación, asesoramiento y seguimiento de la población con deficiencias y dificultad de adaptación. Si bien, no es hasta 1980 cuando estos equipos empiezan a funcionar, de modo experimental y contratados por el Instituto Nacional de Educación Especial en los centros educativos. "Con ello, los primeros Trabajadores Sociales acceden al Sistema desde estos servicios externos, aunque ya estaban presentes en algunos Centros de Educación Especial y dependencia pública." (CONSEJO GENERAL DE TRABAJO SOCIAL, 2011, p. 59). Posteriormente, en la década de 1980 a 1990, se produjeron diversos fenómenos que consolidaron la figura del Trabajo Social en el sistema educativo español: arraigo del perfil del Trabajo Social en el ámbito educativo como parte del personal laboral de los Equipos Multiprofesionales; amplio desarrollo normativo sobre la Educación Especial y puesta en marcha del Programa de Integración del alumnado con necesidades especiales.

Entre los años 1990 y 2002, tuvo lugar el desarrollo de diferentes normativas y acontecimientos que reportaron un desarrollo significativo. Destacar la Ley Orgánica de Ordenación General del Sistema Educativo (ESPAÑA, 1990) y La Ley Orgánica de la Calidad de la Educación (ESPAÑA, 2002), permitieron la delimitación de las atribuciones y funciones del perfil profesional del Trabajo Social en este ámbito. Mediante las Instrucciones del 26 de febrero de la Dirección General de Renovación Pedagógica (DIRECCIÓN GENERAL DE RENOVACIÓN PEDAGÓGICA, 1990), se estableció el funcionamiento de los Departamentos de Orientación de los Centros de Educación Especial, por el que el personal de Trabajo Social en el ámbito escolar debía favorecer el desarrollo integral del alumnado en colaboración con el resto de 
profesionales. En 1992, la Orden de 9 de diciembre, por la que se regulaba la composición, estructura y funciones de los Equipos de Orientación Educativa y Psicopedagógica (ESPAÑA, 1992), se encargó de unificar los Servicios de Orientación Escolar y Vocacional y los Equipos Multiprofesionales (CONSEJO GENERAL DE TRABAJO SOCIAL, 2011).

La Ley Orgánica 10/2002 de 23 de diciembre de la Calidad de Educación (ESPAÑA, 2002), prioriza la valoración y atención educativa al alumnado con necesidades educativas especiales y al establecimiento de recursos como las aulas especializadas en centros ordinarios. Asimismo, La Ley Orgánica de Educación 2/2006 de 3 de mayo (ESPAÑA, 2006), donde además de recoger los principios fundamentales del sistema educativo, como la equidad o educación de calidad, en su Preámbulo ya indica que el sistema educativo ha de contar con los recursos necesarios que aseguren la inclusión, integración y desarrollo de alumnos que requieran de apoyo educativo. Por último, la Ley Orgánica 8/2013, de 9 de diciembre, para la mejora de la calidad educativa (ESPAÑA, 2013), establece la necesidad de actualizar el sistema educativo. Su pretensión es adaptar dicho sistema a los cambios y exigencias de la sociedad actual, evitando las situaciones de exclusión y, por ende, mediante una participación efectiva de todos los agentes en los contextos social, cultural, familiar y económico, procurar la inclusión, integración, exigencia y calidad educativa en el marco de igualdad de oportunidades. Por tanto, la figura del Trabajo Social, que en sus inicios se incardinaba en los Centros de Educación Especial, ha ido evolucionando conforme a las diversas exigencias sociales y del alumnado, en el marco del Estado Español, y según Castro y Pérez (2017, p. 4) constatan:

La pluralidad de circunstancias contempladas en las distintas etapas históricas, evidencian la importante labor del Trabajo Social frente al reto de detectar necesidades de cada época, adaptar su intervención a los problemas coyunturales, conocer de primera mano la situación de las familias y de la persona con la finalidad de mediar y resolver múltiples situaciones que permitan una eficaz intervención en el contexto familiar.

El Trabajo Social Educativo pretende desarrollar al máximo las capacidades del alumnado, así como mediar entre éstos y los diferentes contextos que lo rodean (social, familiar y escolar). Con ello se intenta convertir la educación en el instrumento más idóneo para el desarrollo de los sujetos en todos los ámbitos de la vida, así como una herramienta consustancial a la evolución de un pais y su cohesión social. Siguiendo a Puyol y Hernández (2009, p. 99):

[..] en lo que se refiere a los centros educativos, el Trabajo Social tiene un papel importante en [...] en temas como el absentismo y el fracaso educativo, la integración de inmigrantes y colectivos desfavorecidos, la detección temprana de malos tratos en el ámbito familiar, la mejora del clima 
de convivencia escolar, la intervención en situaciones de acoso escolar o la estimulación a la participación activa.

Según Castro y Pérez (2017, p. 9) su actuación permite:

[..] mejorar los estilos y estrategias de afrontamiento a toda la comunidad educativa, a través de la información y formación de programas de detección-prevención y de actividades escolares concretas, consigue generar pautas de buena convivencia, así como para prevenir posibles alteraciones conflictivas de tipo emocional ligadas a los cambios sociales, fisicos y psicológicos de la persona.

Las actuaciones de estos/as profesionales pretenden prevenir y paliar las problemáticas que se presentan en los sistemas alumno-familia-escuela, por lo que sus intervenciones van desde el nivel microsocial al trabajo social grupal y/o comunitario. Es, por tanto, consustancial a la educación, la figura y funciones desempeñadas por los/as profesionales del Trabajo social.

\section{EL SISTEMA EDUCATIUO ESPAÑOL}

El sistema educativo español es reconocido como derecho universal dado que es un servicio público que se sustenta sobre el derecho de la ciudadanía a recibir una educación de calidad, basado todo ello en el principio de igualdad de oportunidades, siendo uno de los pilares básicos de nuestro Estado social democrático y de derecho. En esta línea se entiende por sistema educativo español:

\footnotetext{
El conjunto de Administraciones educativas, profesionales de la educación y otros agentes, públicos y privados, que desarrollan funciones de regulación, de financiación o de prestación de servicios para el ejercicio del derecho a la educación en España, y los titulares de este derecho, así como el conjunto de relaciones, estructuras, medidas y acciones que se implementan para prestarlo. (ESPAÑA, 2013, p. 97867).
}

Según se establece en la Ley Orgánica para la Mejora de la Calidad Educativa, por la que se modifica la Ley Orgánica de Educación, “[...] el reto de una sociedad democrática es crear las condiciones para que todos los alumnos y alumnas puedan adquirir y expresar 
sus talentos, [...] el compromiso con una educación de calidad como soporte de la igualdad y la justicia social." (ESPAÑA, 2013, p. 7858).

La finalidad del actual sistema educativo debe ser contribuir el desarrollo integral y personalizado de todos y cada uno de los alumnos con respecto al conjunto de los objetivos que se persigue en cada una de las etapas educativas. Las "Administraciones educativas dispondrán del profesorado de las especialidades correspondientes y de profesionales cualificados, así como de los medios y materiales precisos para la adecuada atención del alumnado con necesidad específica de apoyo educativo (ar.72.1)." (ESPAÑA, 2006). Es aquí donde se reconoce, entre otros, la presencia de la figura del/a trabajador/a social en el ámbito educativo, con objeto de prestar unos servicios de calidad adaptados al amplio espectro de necesidades educativas del alumnado que requiera de una atención educativa diferente a la ordinaria, por presentar dificultades específicas de aprendizaje, tales como: Trastorno por Déficit de Atención e Hiperactividad, por sus altas capacidades intelectuales, por haberse incorporado tarde al sistema educativo o por condiciones personaleso de historia escolar, para que pueda alcanzar el máximo desarrollo posible de sus capacidades personales y, en todo caso, los objetivos establecidos con carácter general para todo el alumnado.

Un concepto que ha evolucionado progresivamente hasta enmarcarse en los principios de normalización e inclusión, envuelto por la accesibilidad universal.

\section{MÉTODO E INSTRUMENTOS DE INUESTIGACIÓN}

El objetivo general de la investigación que orienta el presente artículo es conocer la realidad del Trabajo Social en el ámbito educativo y concretamente: los centros/servicios del ámbito educativo en el que se encuentran desempeñando su labor los/las profesionales del Trabajo Social, las necesidades educativas de los/as niños/as que atiende y las funciones que desarrolla el/la profesional con los/as niños/as con necesidades educativas especiales.

Las dimensiones de análisis para conocer los principales centros/servicios del ámbito educativo en el que se encuentran los/as profesionales y la tipología de necesidades que se atiende son: nivel en el que desempeñan su labor profesional; centro/servicio en el que trabaja; dependencia jurídica del centro/servicio en el que se encuentra; ámbito de actuación del centro/servicio; necesidades que atiende el centro/servicio en el que trabaja. Respecto al objeto de delimitar las funciones que son desempeñadas por estos/ as profesionales en el ámbito educativo ante situaciones de necesidades especiales, se han considerado las siguientes variables de investigación: funciones que desempeñan en su puesto de trabajo; principales funciones realizadas habitualmente en el centro/servicio; 
funciones consideradas más relevantes en el desempeño de su labor profesional; grado de pertinencia de las funciones.

La metodología de esta investigación es mixta (cuantitativa y cualitativa) y la podemos secuenciar en dos fases: la primera ha consistido en realizar un análisis documental de la normativa vigente en materia de educación, a nivel estatal y autonómico, para la elaboración del marco referencial y la construcción del cuestionario. Una vez obtenidos los datos de los cuestionarios se ha procedido a mantener las entrevistas a expertos y reunión con grupo de profesionales del Trabajo Social del ámbito educativo. El trabajo de campo se inició en enero de 2018 mediante la administración del cuestionario a profesionales a través de los colegios profesionales de las provincias de Alicante, Almería y Murcia. El trabajo de campo concluyó en junio de 2018 tras finalizar las entrevistas con expertos y grupo focal. Se ha utilizado un muestreo no probabilístico, al desconocer la totalidad de profesionales del Trabajo Social en el ámbito educativo por la inexistencia de censo identificado. A través de los Colegios Profesionales de las respectivas provincias se ha concretado la muestra integrada por los/as profesionales del Trabajo Social que se encuentra desempeñando sus labores profesionales en el ámbito educativo. Igualmente, se ha recurrido al muestreo mediante la técnica de bola de nieve, pues a través de la localización de determinados/as profesionales que han colaborado en la investigación, se ha podido contactar y obtener la colaboración de otras personas profesionales de relevancia para el desarrollo del análisis del presente proyecto. Las personas participantes han sido un total de 30 profesionales de Trabajo Social del ámbito educativo (entrevistas y grupo de expertos).

La encuesta administrada ha consistido en un cuestionario estandarizado y anónimo, con respuestas preestablecidas y una pregunta abierta. Este instrumento ha constado de 19 preguntas, dividido en cuatro bloques diferenciados: perfil social y formativo de los profesionales; centros y servicios donde desarrollan su labor profesional; funciones y expectativas y dificultades percibidas por los profesionales.

En la entrevista con grupo de expertos/as participaron un total de 10 profesionales que desempeñan su labor en los Servicios de Apoyo Psicopedagógico. Así mismo, se han mantenido entrevistas con expertos/as una vez recogidos los datos con el fin de favorecer sula participación en el análisis de los datos derivados de la investigación.

Para el tratamiento de los datos, se ha utilizado la herramienta de Google Formularios, a través de la cual se ha elaborado el cuestionario y obtenido los datos del mismo. En un segundo momento, se han procesado y analizado los datos a través de Excel, mediante la elaboración de tablas y gráficos de barras. 


\section{ANÁLISIS DE LOS CONTEXTOS DE INTERUENCIÓN AUTONÓMICA}

El análisis comparativo de la normativa, administraciones, competencias y funciones según las diferentes provincias estudiadas nos permite identificar similitudes y diferencias en cuanto a los centros/servicios y funciones de los profesionales del Trabajo Social en el ámbito educativo en el Sureste de España (Murcia, Almería y Alicante).

En primer lugar, respecto a los Equipos de Orientación Educativa y Psicopedagógica en la Comunidad Autónoma de la Región de Murcia, los clasifican en: Equipos de Orientación Educativa y Psicopedagógica de Sector, específicos y de atención temprana. Los/as trabajadores/as sociales dentro de estos equipos, como orientadores/as, se incardinan como Profesotres Técnicos de Servicios a la Comunidad y realizan sus funciones de apoyo en los centros educativos de primaria, secundaria y atención temprana. Tienen como objeto la valoración e intervención en los contextos familiar, escolar y social. En Alicante, las funciones y figura del Trabajo Social equiparable a estos equipos de orientación se traducen en los Servicios psicopedagógicos educativos y gabinetes psicopedagógicos escolares de ámbito municipal. Éstos prestan sus servicios en los centros educativos de Educación Infantil, Primaria, Secundaria y Educación Especial. Se adscriben a los centros educativos de cada sector según las necesidades educativas y sociales concretas de cada centro y su personal no forma parte de los centros educativos. A pesar de la diferenciación en sus denominaciones, estos servicios psicopedagógicos coinciden con las funciones previstas para los/as trabajadores sociales, aun no siendo enunciadas de manera exacta. Estos equipos multiprofesionales de orientación, se organizan y denominan en Andalucía, y más concretamente Almería, como Equipo Técnico Provincial para la Orientación Educativa y Profesional, Equipo de Orientación Educativa y Equipo de Orientación Educativa Especializado. Dichos equipos desempeñan sus funciones especializadas en las áreas de orientación educativa, atención a los alumnos y alumnas con necesidades especiales, compensación educativa y apoyo a la acción tutorial del profesorado y actúan en el conjunto de los centros de una zona educativa, atendiendo a los niveles de Educación Infantil y Primaria. Si bien, en la etapa de secundaria, los centros cuentan con un departamento de orientación, que desarrolla las funciones propias de orientación, conformado por un orientador/a, los tutores/as de cada curso y el jefe/a de estudios del Centro. En Alicante la figura del Trabajo Social se prevé también en los unidades educativas terapéuticas / hospitales de día infantil y adolescente, que se consolidan como un escenario de intervención en el ámbito educativo y sanitario para el personal de Trabajo Social, como centros de recursos de atención integral, multiprofesional y especializada, para dar respuesta a las necesidades del alumnado con enfermedades que impidan su adecuada escolarización. 
En segundo lugar, la Atención Temprana está regulada en las tres comunidades autónomas estudiadas, los/as profesionales del Trabajo Social no sólo se incardinan en los Centros de Desarrollo Infantil y Atención Temprana como agentes que dependen de los Servicios Sociales, sino que también los Equipos de Orientación Educativa y Psicopedagógica, los Servicios psicopedagógicos y Equipos de Orientación Especializada-desarrollan sus funciones en este nivel y en colaboración con dichos centros. Los Centros de Educación Especial, en la Región de Murcia quedan registrados como centros de recursos que debiendo estar a disposición de los centros ordinarios, se dirigen a atender al alumnado con necesidades especiales. Éstos, que se configuran como centros de empleabilidad para los/ as profesionales del Trabajo Social, forman parte de su personal no docente, y sus funciones están reguladas de acuerdo a la Orden de 2003 por la que se determina la jornada, horario especial y funciones del Personal de Atención Educativa Complementaria y otro personal no docente que presta servicios en los centros docentes. Si bien, en Alicante se especifica que la atención desde el Trabajo Social será facilitada por los servicios psicopedagógicos del sector correspondiente en los centros de educación especial dependientes de la Administración.

En conclusión, observamos que, si bien existen ciertas diferenciaciones entre los centros y servicios en los que se integran los/as profesionales del Trabajo Social respecto a denominación o niveles que abarcan, en cuanto a contenido son similares. Asimismo, las funciones que han de desempeñar los profesionales en los diferentes equipos de los centros/ servicios presentados en esta investigación, no varían sustancialmente de una Comunidad Autónoma a otra, aunque en cada una de ellas existe una regulación específica a tal efecto.

\section{DESCRIPCIÓN Y ANALISIS DE RESULTADOS}

\subsection{PERFIL SOCIAL Y FORMATIUO DE LOS TRABAJADORES SOCIALES QUE SE ENCUENTRAN EN EL ÁMBITO}

El porcentaje de profesionales mujeres es notablemente superior $(81 \%$ ) al de varones (19\%). Respecto a la edad, el mayor porcentaje se sitúa en los tramos de edad comprendido entre los 41 y 50 años (31,8\%), seguido por los grupos de entre 26 a 35 años y de 51 a 55 años. En cuanto a la formación, los profesionales que disponen de otra titulación diferente a la de Trabajo Social asciende al 63,5\% frente al 31,9\% que no dispone de otra titulación. De las personas que ostentan alguna titulación diferente a la de Trabajo Social, un $50 \%$. Respecto a la jornada laboral que desarrollan, el 81,8 trabaja a jornada completa. 


\subsection{CENTROS/ SERUICIOS EN LOS QUE DESEMPEÑAN SU LABOR PROFESIONAL}

En cuanto a los niveles educativos en los que desempeñan su labor profesional, indicar que preferentemente se encuentran en educación primaria, concretamente el $45,5 \%$. La investigación muestra que los equipos de orientación educativa de Sector y Específicos son dos de los espacios en los que más trabajadoras/es sociales desempeñan su labor profesional, seguido de los Centros de Educación Especial. Los centros son fundamentalmente dependientes de la Administración Pública (63,6\%). Respecto al ámbito de actuación del centro/ servicio en el que desempeñan sus funciones los profesionales es, por lo general, municipal.

Conforme se muestra en el Gráfico siguiente (1), las principales necesidades que atiende, concretamente:
a) absentismo y abandono escolar 14 (63,6\%);
b) orientación familiar 13 (59\%);
c) necesidades educativas especiales $12(54,5 \%)$;
d) atención a la diversidad $11(50 \%)$.

Gráfico 1 - Necesidades a las que se dirige el centro/servicio

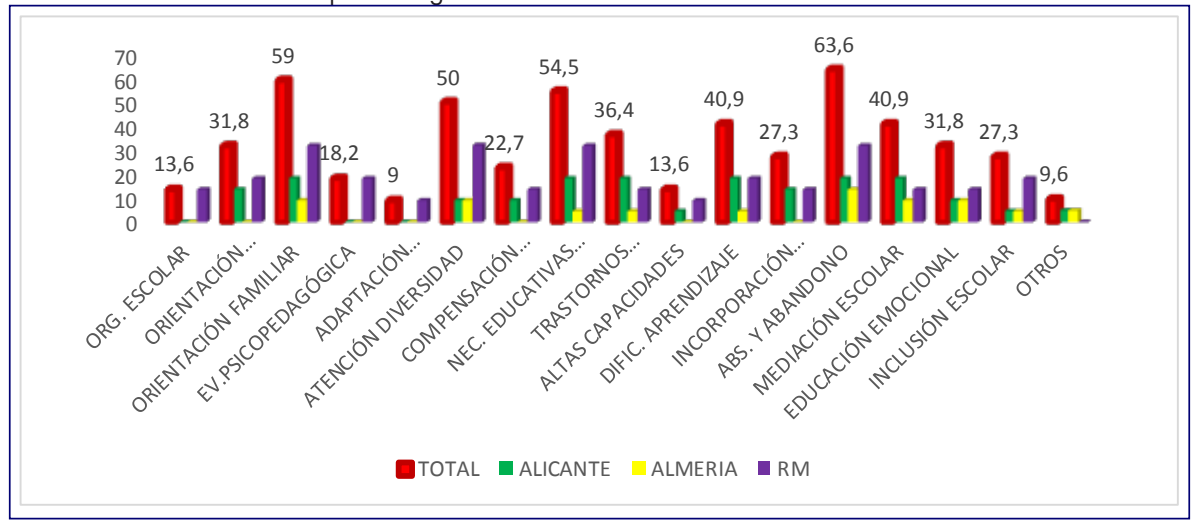

Fuente: los autores.

Las necesidades sociales que se atiende preferentemente por provincias y orden de importancia son: 
a) Provincia de Alicante: orientación familiar, necesidades educativas especiales, trastornos de la conducta, dificultades de aprendizaje, absentismo y abandono escolar y, por último, mediación escolar, todas seleccionadas por las personas participantes;

b) Región de Murcia, las necesidades más importantes son: atención a la diversidad, necesidades educativas especiales, orientación familiar y absentismo y abandono escolar;

c) Provincia de Almeria: absentismo, abandono escolar, necesidades de orientación familiar, atención a la diversidad, necesidades educativas especiales y educación emocional.

Por tanto, las necesidades por las que son utilizados los centros/servicios por los/as niños/as y sus familias son principalmente: orientación familiar, absentismo y abandono escolar y necesidades educativas especiales.

En cuanto a las funciones profesionales principales desempeñadas para la atención de las necesidades de los/as niños/as destacan:
a) prevenir situaciones de conflicto, problemas de adaptación y conductas disruptivas, así como atender y resolver situaciones individuales: absentismo, bajo rendimiento, problemas de relación y comunicación, etc. con el 66,6\%;
b) realizar y participar en las tareas de orientación familiar con el 66,6\%;
c) colaborar con las instituciones en las intervenciones que requieran derivación, intervención conjunta $y / o$ coordinación con los servicios existentes para dar respuestas globales e integrales a las necesidades del alumnado, por el 66,6\%;
d) favorecer las relaciones familiares entre si y de estas con el centro con el $61,9 \%$.

En conclusión, según lo indicado por los/as trabajadores/as sociales, las funciones más desempeñadas en su puesto de trabajo son:
a) prevenir situaciones de conflicto;
b) problemas de adaptación y conductas disruptivas; 

c) atender y resolver situaciones individuales;
d) realizar y participar en tareas de orientación familiar;
e) colaboración, intervención conjunta y coordinación con otros servicios para dar respuestas globales e integrales;
f) mejorar las relaciones familiares entre sí y de éstas con el centro.

Las funciones desempeñadas con mayor habitualidad, conforme nos muestra el Gráfico 2, (las más seleccionadas por las profesionales de entre las 17 incluidas en el cuestionario), son:

a) Fl, prevenir situaciones de inadaptación y delincuencia juvenil, así como atender y resolver situaciones individuales: absentismo, bajo rendimiento problemas de relación y comunicación, etc., indicada por $54,4 \%$;

b) F2, detectar desajustes familiares: malos tratos, deficiencias alimentarias, desajustes emocionales, entre otros, señalada por el $54,4 \%$.

Gráfico 2 - Funciones desempeñadas con mayor habitualidad

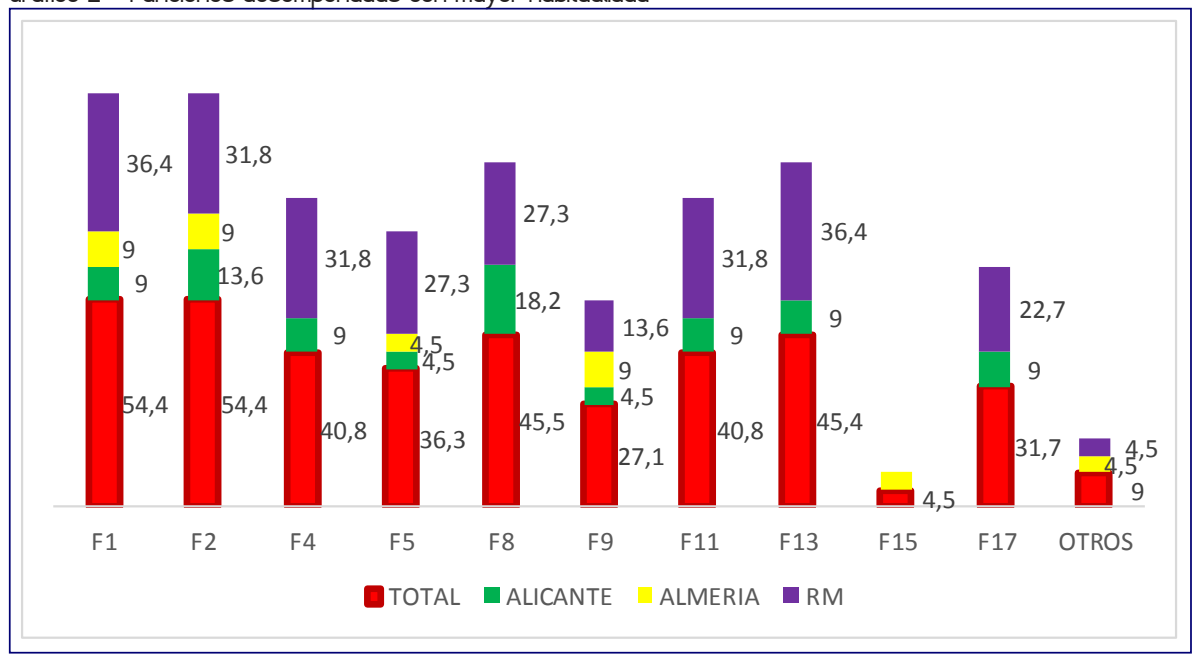

Fuente: los autores. 
En conclusión, las funciones que desempeñan con más habitualidad son aquellas relacionadas con la prevención de problemáticas individuales de los/as niños/as, así como las vinculadas con problemáticas familiares.

Respecto a las funciones consideradas por los profesionales como más relevantes por parte de los/as trabajadores en el ámbito educativo son:

a) F2, detectar desajustes familiares: malos tratos, deficiencias alimentarias, desajustes emocionales, etc, , con el 63,5\%;

b) F13, orientar a las familias para que puedan resolver sus dificultades relacionales, con el 59,1\%;

c) Fl, prevenir situaciones de conflicto, problemas de adaptación y conductas disruptivas, así como atender y resolver situaciones individuales: absentismo, bajo rendimiento, problemas de relación y comunicación con el 54,5\%;

d) F4, comunicar y proporcionar al equipo y centro de educación la información necesaria acerca del diagnóstico socio familiar del alumnado, con un $50 \%$.

Gráfico 3 - Funciones percibidas por los profesionales como las más relevantes

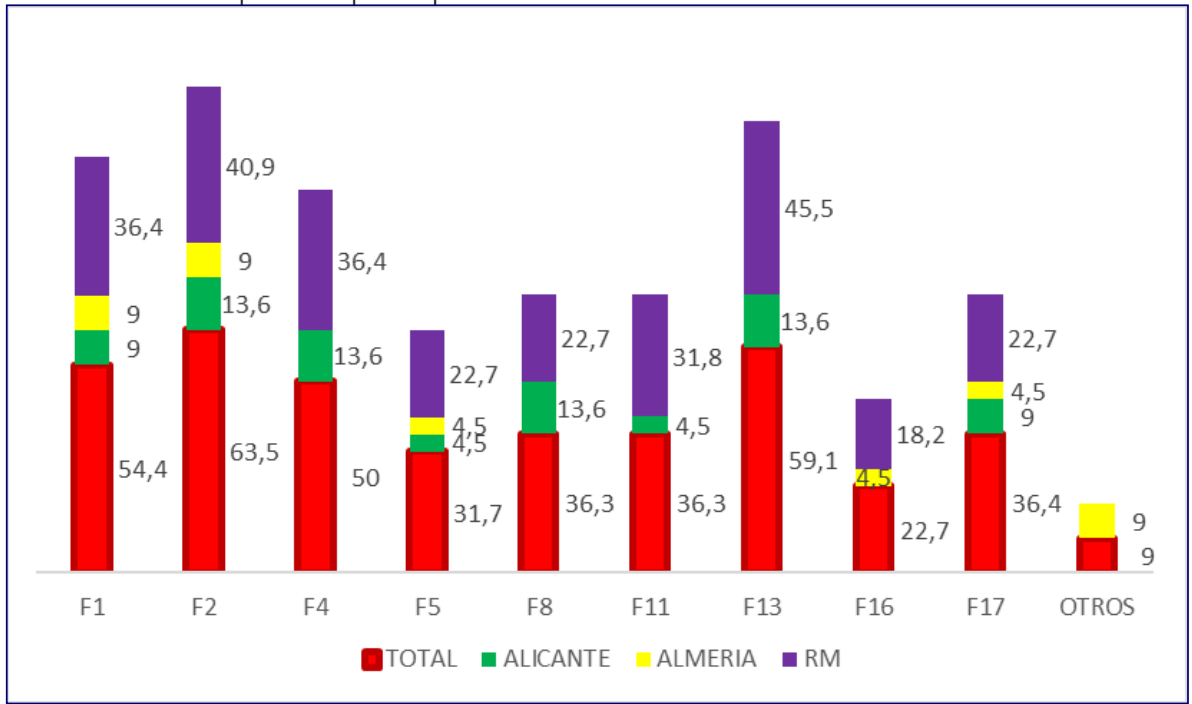

Fuente: los autores. 
En esta línea, a la cuestión relativa al "żpor qué el Trabajo Social en el ámbito educativo se focaliza en el entorno familiar del alumno? ", intenta dar respuesta a porqué las funciones consideradas más relevantes están relacionadas con el entorno familiar:

[...] no se considera directamente dentro de la comunidad educativa. Es como alguien ajeno a la escuela. Por eso se trabaja desarrollando proyectos que no son propiamente escolares, es decir, tareas que emanan directamente de la educación formal. (Entrevista 1 Alicante).

La influencia del entorno social y familiar en las personas, en nuestro caso alumnos, es innegable por lo que está claro que cuando existen disfunciones en estos entornos debemos de actuar. El hecho de actuar desde educación y no desde servicios sociales (en primera instancia), hace que la intervención sea percibida como menos invasiva por parte de los padres que en muchas ocasiones no quieren que estos servicios de protección intervengan. También es importante la labor que realizamos con las familias desde la orientación, ya que en muchas ocasiones nos encontramos con sistemas cerrados que no buscan ayuda, pero las orientaciones que desde el colegio les podemos dar son bien recibidas, no debemos de olvidar que los centros escolares nos ofrecen un balcón de observación privilegiado para poder detectar disfunciones familiares que desde otros ámbitos pasaría desapercibido. (Entrevista 2 Almería).

Es el único profesional dentro del ámbito educativo especializado en atender las necesidades socio-familiares; los centros educativos son el marco idóneo para detectar situaciones de riesgo para los menores y debe ser prioritario la intervención en este aspecto. Se atiende al alumno a nivel integral, actuando siempre vinculando las necesidades personales con las educativas. (Entrevista 3 Región de Murcia) (informaciones verbales).

Tal y como se observa, las funciones consideradas como más relevantes por parte de las personas participantes en la investigación están directamente relacionadas con el entorno familiar de los/as niños/as, lo que se constata al comprobar las funciones que han sido elegidas por la mayoría de profesionales. Asimismo, el contexto educativo constituye un marco ideal para intervenir de manera integral y detectar situaciones de riesgo o disfunciones familiares.

En conclusión, en lo relativo a las funciones, según la explotación de los datos procedentes de los cuestionarios, tanto por su desempeño, habitualidad y relevancia en el puesto de trabajo, destacan aquellas: dirigidas a la prevención y atención de problemáticas individuales de los menores; relacionadas con la familia, la orientación familiar o la elaboración del diagnóstico social y familiar; asi como la colaboración, coordinación e intervención con otros servicios y los profesionales del propio centro para dar respuestas globales e integrales. En cuanto a la pertinencia de las funciones, se observa que hay opiniones dispares; mientras unos/as consideran que realizan las funciones que deben, otros/as estiman que deberían realizar otras con mayor relevancia e indicando limitaciones institucionales en su desempeño o que no se corresponden con su puesto de trabajo. 


\title{
5.3 EXPECTATIUAS Y DIFICULTADES PARA EL TRABAJO SOCIAL EN EL ÁMBITO EDUCATIUO
}

Según el criterio de las personas participantes, el nivel de integración profesional en el ámbito educativo es bajo, en un $45,5 \%$ y muy bajo en el $31,8 \%$. Respecto a los motivos de esta baja incorporación, las personas participantes indican.

\begin{abstract}
No ha habido nunca un convencimiento en los gobernantes y directores de las diferentes consejerías de educación. Existen pocos profesionales no maestros en la escuela española. Además, los trabajadores sociales, en cierto sentido están siendo solapados por otros profesionales, por ejemplo, los psicólogos. (Entrevista 4 Almería).

Por el propio desconocimiento que los politicos tienen sobre las funciones que realizan los trabajadores sociales de educación. (Entrevista 5 Alicante). Es necesaria la creación de más plazas, tanto en EOEPS como en IES. Las existentes son insuficientes. La Administración Pública tiene que dar respuesta a esta necesidad. (Entrevista 6 Región de Murcia) (informaciones verbales).
\end{abstract}

Por consiguiente, se considera que el desconocimiento por parte de agentes políticos y directivos es causa de este suceso, recalcando la necesidad de creación de más plazas de trabajo en los equipos donde desarrollan sus funciones por parte de la Administración Pública. Esta afirmación se constata cuando se le pregunta acerca del (re) conocimiento de la figura del Trabajo social en el ámbito educativo donde el $100 \%$ de los participantes afirman que no es conocida por la sociedad, cuestión que se constata también en las entrevistas y grupo de expertos como se evidencia a continuación:

\footnotetext{
No es conocida por la población porque no existe. Llevamos además unos años que estamos contemplando el encerramiento y la identificación de los trabajadores sociales en los servicios sociales. Se han secuestrado o auto-secuestrado en algunos casos en los despachos y en un trabajo de gestión. (Entrevista 7 Almería).

Creemos que hay tres: 1. Dentro del ámbito educativo los profesionales que atienden de forma externa a los centros son en su gran mayoría orientadores, lo que hace que nuestra figura quede invisibilizada. En ocasiones hasta por los propios docentes y orientadores; 2.- Cuando nos presentamos en los centros bien a los docentes, bien a las familias, en la presentación se escucha "trabajador social" pero el apellido de educación pasa desapercibido, por lo que los interlocutores directamente nos reasignan a servicios sociales, sistema público mucho más conocido por todos; 3.El número tan escaso de trabajadores sociales en educación hace que sea poco relevante a nivel legislativo, a nivel de literatura científica, poca
} 
visibilización por parte de las universidades, escasa apuesta municipal para su contratación, ... Desconocimiento = invisibilización. (Entrevista 8 Alicante). La inclusión de este perfil profesional en el ámbito educativo ha sido muy tardía [...] la presencia es reducida. Además, no en todas las CCAA está presente este perfil en todas las etapas educativas (por ejemplo en Andalucía no existe en la etapa de Secundaria: Departamentos de Orientacion). Además, en muchos casos no son Trabajadores Sociales los que desempeñan la labor profesional sino PTSCSs. (Grupo de Expertos) (informaciones verbales).

Se constata que existe la percepción por parte de los/as trabajadores/as sociales que su labor profesional en educación es totalmente desconocida por la sociedad. Esto puede deberse principalmente a la identificación de los/as trabajadoras sociales con el Sistema de Servicios Sociales y/o por la escasa presencia e invisibilización de estas/os profesionales en el ámbito de la educación como ha quedado constatado.

En cuanto a los contextos donde estiman que existen mayores posibilidades de incorporación laboral, tal y como muestra la siguiente Gráfica (4) los equipos de orientación educativa de Sector constituyen el 50\%, y los centros de educación especial el 40,9\%.

Gráfico 4 - Centros/servicios con más posibilidades de empleo en educación para el Trabajo Social

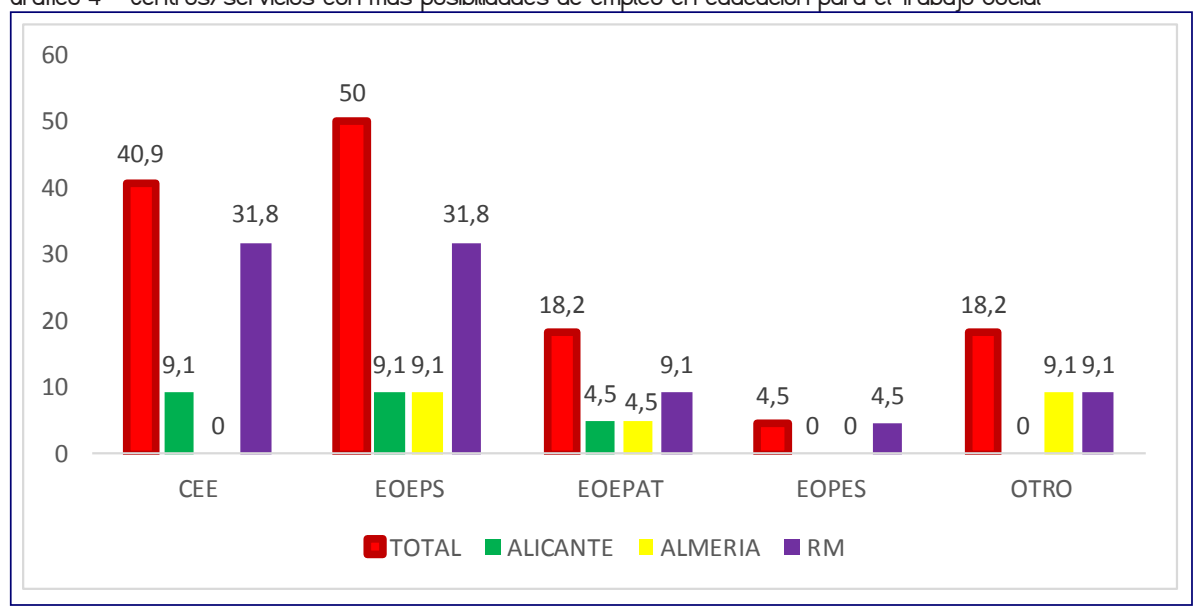

Fuente: los autores.

Las entrevistas y grupo de expertos confirman la información derivada de los cuestionarios, conforme se evidencia a continuación:

Si, y no solamente en los dependientes de las comunidades autónomas, sino también en las dependencias municipales y por su puesto en los puestos 
estructurales de las Consellerias de Educación junto con los servicios de prevención de la violencia y absentismo escolar. (Entrevista 1 Alicante).

Por supuesto. Deben aumentar las ratios por centros (tanto en Primaria como en Secundaria). Y crear donde no existan. (Entrevista 3 Región de Murcia) (informaciones verbales).

\begin{abstract}
En cuanto a las expectativas de futuro profesional en el ámbito educativo los expertos informan:
\end{abstract}

Las expectativas, yo las veo, muy muy escasas. Motivos: no se ha descubierto la necesidad; restricciones económicas para la realización de nuevas contrataciones: falta de aclarar las competencias. (Entrevista 1 Alicante).

Desde el grupo de Alicante llevamos mucho tiempo por una ampliación de la plantilla y que ésta sea provista desde un temario específico de educación, no por admisión desde función pública, pero no hemos conseguido nada, por lo que nuestra percepción siendo realistas es que sus expectativas de futuro no son muy esperanzadoras. (Entrevista 2 Almería).

Con respecto al empleo, crear oferta de Empleo Público. Con respecto al ámbito de actuación, en el caso de Primaria se adecúa y corresponde con las funciones del perfil profesional, así como el estar enmarcado dentro de la interdisciplinariedad. La precariedad es el número elevado de centros por sector en relación al número de profesionales. (Grupo de Expertos) (informaciones verbales).

\begin{abstract}
En cuanto a las principales dificultades que existen para consolidar la figura del trabajo social en el ámbito educativo:
\end{abstract}

\begin{abstract}
El reconocimiento de nuestro trabajo y apuesta verdadera por equipos directivos de centros escolares (tanto de infantil y primaria como de secundaria), directores de los servicios psicopedagógicos escolares y por su puesto de la Inspección, de tal manera que puedan hacer ver a los políticos de la necesidad existente de esta figura para trabajar desde la prevención. (Entrevista 10 Alicante).

Se considera una profesión de segunda para la atención de personas necesitadas, sobre todo económicamente. No se conocen dimensiones del trabajo social como: la investigación o el diagnostico; el trabajo social de grupos; el trabajo con una metodología específica, etc. (Entrevista 11 Almería). La principal dificultad en mi opinión es la ausencia de profesionales, no se puede consolidar una figura que no está presente en muchos centros educativos. En los que sí existe, la figura está consolidada. (Entrevista 3 Región de Murcia) (informaciones verbales).
\end{abstract}

En lo relativo a las fortalezas del Trabajo Social para/con el ámbito educativo en la actualidad: 
Pensamos que la orientación familiar dentro de cualquier problemática de las destacadas en los resultados es y creemos que seguirán siendo la fortaleza de nuestras intervenciones [...] También la mediación sería una de las herramientas en las que tendriamos que focalizar nuestras fortalezas. (Entrevista 10 Alicante).

Según en qué nivel se sitúe: a nivel de planificación, el Trabajo Social, puede aportar en la creación de nuevas vías de intervención socioeducativa; a nivel de metodología, una metodología activa, participativa a través de la cual se implican los diferentes sectores de la comunidad educativa; a nivel observatorio de educación (interesante a nivel municipal) detección de nuevos campos de intervención; a nivel de trabajo con las familias mediante desarrollo programas de dinamización a las familias y de integración familiar. Además, es una ayuda para poner "los pies en la tierra", en ocasiones los docentes se pueden encontrar fuera de la realidad [...] aportando al mismo la dimensión y compromiso social por el cambio. Analiza y diagnostica incipientes formaciones de ghettos. Y trabaja la inclusión y la atención a la diversidad, etc. (Entrevista 11 Almería).

El Trabajo Social es una disciplina especializada en poder abordar las funciones específicas que requiere el trabajo socioeducativo en los Centros. Creo que, en este sentido, esta Titulación debería ser requisito en el acceso a las plazas en este ámbito. (Entrevista 14 Región de Murcia) (informaciones verbales).

En conclusión, las principales dificultades que se encuentra el Trabajo Social en el ámbito educativo son: el escaso reconocimiento por parte de agentes clave de la necesidad de trabadores sociales y de la relevancia de las funciones que realizan en el ámbito educativo. Todo ello conlleva a la ausencia de profesionales en los pertinentes centros y servicios. Si bien, entre las fortalezas que esta disciplina reporta a la educación, se pueden destacar varias: su capacidad para intervenir y mediar entre los diferentes contextos, la participación activa, detección precoz de problemáticas o nuevas necesidades, el compromiso social por el cambio, o la formación específica para actuar en este campo.

Respecto a expectativas según los resultados obtenidos: los/as profesionales consideran que el nivel de empleabilidad en este ámbito es bajo o muy bajo; la figura deV la trabajador/a social en educación, además de escasa, es desconocida por la sociedad; las expectativas de futuro en el sistema educativo para estas/os profesionales no son muy alentadoras; los equipos de orientación de Sector y Específicos y los centros de educación especial, son los centros/servicios que se consideran con una mayor oportunidad de empleo para el Trabajo Social; las principales dificultades para los/as profesionales están relacionadas con la falta de reconocimiento de sus funciones y labor profesional, mientras que las fortalezas van ligadas a los conocimientos, herramientas y metodologías propias del Trabajo Social. 


\section{DISCUSIÓN}

El perfil profesional que desempeña su labor en el ámbito educativo es mayoritariamente femenino. Esto no supone sorpresa alguna, ya que el Trabajo Social viene siendo desde sus inicios una profesión principalmente compuesta por mujeres. Este dato coincide plenamente con el II Informe sobre los Servicios Sociales en España (LIMA, 2015) expone el cual el $83 \%$ de los profesionales del Trabajo Social en España son mujeres. Este dato se aproxima al obtenido en nuestra investigación donde el 81 son mujeres y $19 \%$ varones y coincide con investigaciones al respecto (PASTOR et al., 2017).

Respecto la edad, aun estando presentes en todos los tramos, deja entrever que la mayoría se encuentra entre los 26 y 55 años. Esto podría guardar relación con las dificultades de las personas recién tituladas y aquellas mayores de 55 años de estar incorporadas en el mercado laboral. Si bien, el mayor porcentaje de edad se sitúa entre los 41-50 años junto a los tramos de edad de entre 31-35 y 51-55. Según los datos del II lnforme sobre los Servicios Sociales en España (LIMA, 2015), comprobamos que la mayor parte de las/os trabajadoras/es sociales tienen entre 35 y 54 años, lo que coincide con nuestros resultados. Atendiendo al perfil formativo al menos la mitad ha realizado un máster oficial e incluso un porcentaje relevante dispone de un doctorado. La jornada laboral completa representa el $81,8 \%$ de las personas participantes, en sintonía de igual forma con los datos de la investigación anteriormente mencionada.

Los niveles del ámbito educativo en que las/os trabajadoras/es desempeñan su labor profesional, destaca por encima de todas, la opción de nivel de Infantil, donde se encuentran. Cabe destacar que según los datos obtenidos, el 59,1\% de las/os participantes se encuentran trabajando en algún equipo de orientación interdisciplinares. Otro dato que podemos asimilar al derivado del II Informe sobre los Servicios Sociales en España es respecto al ámbito de actuación del centro/servicio, donde en su gran mayoría son autonómicos y municipales.

En cuanto a las necesidades que atienden las/os profesionales desde los centros/servicios, destaca el absentismo y abandono escolar. Dicha problemática también supone preocupación para el Ministerio de Educación Cultura y Deporte, el cual ha elaborado un Plan para la Reducción del Abandono Educativo Temprano. Esta necesidad va seguida de la orientación e intervención con la familia. El contexto familiar es inherente a las problemáticas y necesidades del alumnado.

Las funciones desempeñadas con mayor habitualidad y que son consideradas como más relevantes por las/os trabajadoras/es sociales del ámbito educativo, son aquellas que conciernen tanto al alumno/a y su problemática, como al contexto familiar y al centro 
educativo. Por tanto, estamos ante un perfil profesional capaz de intervenir simultáneamente en diferentes contextos. Ello propicia la coordinación, colaboración e intervención conjunta entre los diferentes agentes educativos y sociales. Si atendemos a las normativas específicas de cada Comunidad Autónoma para cada uno de los centros/servicios, se han de resaltar dos peculiaridades. En primer lugar, la Orden de 24 de noviembre de 2006 de la Región de Murcia (CONSEJERÍA DE EDUCACIÓN Y CULTURA, 2006), que regula las funciones de los PTSC en los EOEP, y la Resolución del 21 de julio de 2017 de la Comunidad Valenciana (CONSELLERIA DE EDUCACIÓN, INVESTIGACIÓN, CULTURA Y DEPORTE, 2017), permiten afirmar que las funciones de los/as trabajadores/as de los EOEP y los SPE coinciden plenamente en su contenido.

Los resultados permiten afirmar que el Trabajo Socioeducativo se erige como la profesional idónea para atender las necesidades sociofamiliares y detectar situaciones de riesgo, siempre desde una atención integral e interdisciplinar.

Pese a lo anterior, se percibe cierta frustración en el personal de Trabajo Social con experiencia en el ámbito educativo. Como concluye la presente investigación, las expectativas de las normativas de educación (tan ambiciosas y ansiosas por lograr la inclusión y cohesión social) y la realidad socioeconómica, parecen requerir la presencia de personal no docente en el ámbito educativo, especialmente de las/os trabajadoras/es sociales. Sin embargo, la realidad es otra. Es más, las personas participantes han resaltado que la figura del Trabajo Social en el ámbito educativo es plenamente desconocida por la sociedad. La falta de valoración, presencia y expectativas futuras para estas/os profesionales que pretenden converger lo social con lo educativo puede deberse a múltiples factores. Pero es necesario un reconocimiento y compromiso político para que esta situación cambie. Además, la identificación que la sociedad hace del Trabajo Social con el Sistema Público de Servicios Sociales (LIMA; VERDE; PASTOR, 2016; PASTOR et al., 2017) parece ser cada día más un hándicap que una ventaja. Pero ésta visión tan pesimista de la realidad socioeducativa no debe ser más que el motor de empuje para que el Trabajo Social consiga su merecido reconocimiento en el sistema educativo español. Esta disciplina, que se ocupa de lo "social", cuenta con un cuerpo teórico en crecimiento, con herramientas e instrumentos de intervención propias. Además, requiere de unas habilidades profesionales en particular; esto es, un conjunto de fortalezas que erigen al Trabajo Social como profesión idónea para la intervención en el ámbito educativo.

\section{CONCLUSIONES}

El Trabajo Social, como disciplina relativamente joven, se encuentra actualmente en un momento de evolución dentro del sistema educativo español. La figura de este 
perfil profesional, que tiene su origen en los centros de educación especial y que inició su consolidación en el ámbito educativo a partir de los años 70, experimentó un auge durante la etapa de los años 90, extrapolando su presencia a los Equipos de Orientación Educativa, como profesores/as de servicios a la comunidad capaces de intervenir en el ámbito educativo. A tal efecto, e independientemente de las diferentes normativas que han pretendido ocuparse del sistema educativo español a nivel estatal, la regulación de la presencia del Trabajo Social en el ámbito educativo queda en manos de las diferentes Comunidades Autónomas.

Respecto a los contextos territoriales base de esta investigación, concretamente las Comunidades Autónomas de la Región de Murcia, Andalucía (Almería) y Valencia (Alicante), el personal de Trabajo Social está presente en: Equipos de Orientación Educativa y psicopedagógica (en la Región de Murcia), siendo su equivalente en Alicante los servicios psicopedagógicos y gabinetes psicopedagógicos municipales, los Equipos de Orientación Educativa en Almería; Centro de Desarrollo Infantil y Atención Temprana o Equipos Técnicos Provinciales de Atención Temprana; otros. Todos ellos van destinados, primordialmente, a atender la diversidad del alumnado, necesidades especiales, el absentismo escolar y/o la orientación familiar, sin olvidar la necesidad de mediación escolar.

Por tanto, las funciones destacadas por las/os profesionales participantes en la investigación, según desempeño, habitualidad y relevancia han sido: prevenir situaciones de conflicto, problemas de adaptación y conductas disruptivas, así como atender y resolver situaciones individuales: absentismo, bajo rendimiento, problemas de relación y comunicación; realizar y participar en las tareas de orientación familiar; colaborar con las instituciones en las intervenciones que requieran derivación, intervención conjunta y/o coordinación con los servicios existentes para dar respuestas globales e integrales a las necesidades de los alumnos; favorecer las relaciones familiares entre si y de estas con el centro; detectar desajustes familiares: malos tratos, deficiencias alimentarias, desajustes emocionales, entre otros; comunicar y proporcionar al equipo y centro de educación la información necesaria acerca del diagnóstico socio familiar del alumnado. Pero para ello, es necesario unas políticas públicas que doten a los niños y niñas de oportunidades y contextos de igualdad, equidad y participación (PICORNELL; PASTOR, 2016). Los/as niños/as deben ser “[...] destinatários das políticas públicas, más a sua assunção plena como sujeitos políticos peculiares" (SARMENTO; FERNANDES; TOMAS, 2007, p. 190), como ciudadanos en el interior "das organizações e instituições onde as crianças agem." (SARMENT0, 2012, p. 46).

Para concluir, resaltar la percepción negativa por parte de las/os profesionales que han participado en el trabajo, pues todas ellas han puesto de manifiesto tres premisas: existencia de un bajo nivel de empleabilidad para el Trabajo Social en el ámbito educativo; falta de reconocimiento tanto por parte de la sociedad como por los agentes directivos (escolares) y políticos y escasas expectativas de futuro para el Trabajo Social en el ámbito educativo. Estas 
afirmaciones no se traducen en una desvalorización por parte de las propias profesionales. Conocedoras/es de su labor y de las funciones que desempeñan, así como de la realidad social, familiar y educativa en la que están inmersos los/as alumnos/as, son conscientes de que cada día el Trabajo Social debería estar más presente en el ámbito educativo. Todo ello converge en la exigencia de que el personal docente trabaje en colaboración y coordinación con otros/as profesionales.

\section{REFERENCIAS}

CASTRO, C.; PÉREZ, J. El Trabajo Social en el entorno educativo. Barataria. Revista Castellano-Manchega de Ciencias Sociales, n. 22, p. 215-226, 2017. Disponible en: https://doi.org/10.20932/barataria.v0i22.309. Acceso en: 11 feb. 2018.

CONSEJERÍA DE EDUCACIÓN Y CULTURA. Orden de 24 de noviembre de 2006, de la Consejería de Educación y Cultura por la que se dictan instrucciones de funcionamiento de los equipos de orientación educativa y psicopedagógica. Boletín Oficial de la Región de Murcia, Madrid, 24 nov. 2006. Disponible en: https://www.carm.es/web/pagina?IDCONTENID0=4466\&IDTIP0=100\&RASTR0=c148\$m4463. Acceso en: 2 mar. 2018.

CONSEJO GENERAL DE TRABAJO SOCIAL. El trabajo social en el sistema educativo. Madrid: Consejo General de Trabajo Social, 2011. Disponible en: https://www.cgtrabajosociales/publicaciones/el-trabajo-social-en-el-sistema-educativo-digital/13/view. Acceso en: 22 mar. 2018.

CONSELLERIA DE EDUCACIÓN, INVESTIGACIÓN, CULTURA Y DEPORTE. Resolución de 21 de julio de 2017, de la Secretaría Autonómica de Educación e Investigación, por la que se dictan instrucciones para la organización de los servicios psicopedagógicos, escolares y gabinetes psicopedagógicos con escolares autorizados, la elaboración de su plan de actividades y de su memoria durante el curso 2017. Diario Oficial de la Generalitat Valenciana, 21 jul. 2017. Disponible en: http://www.ceice.gva.es/web/ ordenacion-academica/normativa. Acceso en: 6 mayo 2018.

DIRECCIÓN GENERAL DE RENOVACIÓN PEDAGÓGICA. Instrucciones por las que se establece el funcionamiento del Departamento de Orientación Educativa en los centros de educación especial. Madrid: Subdirección General de Educación Especial, 1990. Disponible en: http://die.caib.es/normativa/ pdf/90/1990-02-26_InstruccionsFuncionamentDepartamentOrientacioCentresEducacioEspecial.pdf. Acceso en: 15 jun. 2018.

ESPAÑA. Constitución Española. Cortes Generales de 27 de diciembre de 1978. Madrid, 1978.

ESPAÑA. Ley n. 12, de 2 de julio de 1987. Sobre establecimiento de la gratuidad de los estudios de Bachillerato, Formación Profesional y Artes Aplicadas y Oficios Artísticos en los Centros públicos y la autonomía de gestión económica de los Centros docentes públicos no universitarios. Boletín Oficial del Estado, Madrid, 3 jul. 1987. 
ESPAÑA. Ley Orgánica General del Sistema Educativo, de 3 de octubre de 1990. Boletín Oficial del Estado, Madrid, 4 oct. 1990.

ESPAÑA. Ley Orgánica n. 2, de 3 de mayo de 2006, de Educación. Boletín Oficial del Estado, Madrid, 4 mayo 2006.

ESPAÑA. Ley Orgánica n. 8, de 3 de julio de 1985. Reguladora del Derecho a la Educación. Boletin Oficial del Estado, Madrid, 4 jul. 1985.

ESPAÑA. Ley Orgánica n. 10, de 23 de diciembre de 2002, de Calidad de la Educación. Boletín Oficial del Estado, Madrid, 24 dic. 2002.

ESPAÑA. Ministerio de Educación, Cultura y Deporte. Ley Orgánica n. 8, de 9 de diciembre de 2013. Para la mejora de la calidad educativa. Boletín Oficial del Estado, Madrid, 9 dic. 2013.

ESPAÑA. Ministerio de Educación y Ciencia. Orden de 9 de diciembre de 1992 por la que se regulan la estructura y funciones de los Equipos de Orientación Educativa y Psicopedagógica. Boletín Oficial del Estado, Madrid, 18 dic. 1992.

LIMA, A. I. II Informe de Servicios Sociales en España. Madrid: Consejo General de Trabajo Social de España, 2015.

LIMA, A. I.; VERDE, C.; PASTOR, E. El Trabajo Social en los servicios sociales en España. En: PASTOR SELLER, E. (ed.). Políticas e intervenciones ante los procesos de vulnerabilidad y exclusión de personas y territorios: análisis comparado España-México. Madrid: Dykinson y México: Universidad Nacional Autónoma de México, 2016. p. 173-188.

PASTOR, E. et al. El sistema de servicios sociales y dependencia en España. En: PASTOR SELLER, E. (ed.). Sistemas y políticas de bienestar: una perspectiva internacional. Madrid: Dykinson, 2017. p 111-130.

PICORNELL, A.; PASTOR, E. (coord.). Políticas de inclusión social de la infancia y la adolescencia: una perspectiva internacional. Madrid: Editorial Grupo 5, 2016.

PUYOL, M. B. Y.; HERNÁNDEZ, M. Trabajo social en educación. Qurriculum. Revista de Teoría, Investigación y Práctica Educativa, v. 22, p. 97-117, 2009.

SARMENTO, M. A criança cidadã: vias e encruzilhadas. Imprópria. Política e pensamento crítico, v. 2, p. 45-49, 2012.

SARMENTO, M.; FERNANDES, N. E.; TOMÁS, C. Políticas Públicas e Participação Infantil. Educação, Sociedade e Cultura, v. 25, p. 183-206, 2007.

Enderezos para correspondencia: Universidad de Murcia, Facultad de Trabajo Social, Campus de Espinardo, 30.100, Murcia, Murcia, España; epastor@um.es. 